\title{
Insulin resistance in diabetes mellitus due to chronic liver disease and retroviral infection
}

\author{
Chethana Manjunath · Choodappa Setty Munichoodappa
}

Received: 21 March 2013 / Accepted: 22 November 2013 /Published online: 15 January 2014

(C) Research Society for Study of Diabetes in India 2014

Obese type 2 diabetes mellitus (T2DM) patients are known to have insulin resistance and is often difficult to achieve good glycemic control in them.Insulin resistance also reported in metabolic syndrome, primary disorders of insulin action,lipodystrophies, and certain inherited and acquired disorders [1]. It is also seen in diabetic patients having cirrhosis of liver and retroviral infection [2]. We report a case of a 52 year old business man from rural area, known to have Type $2 \mathrm{DM}$ and Chronic liver disease-Ethanol induced and Hepatitis B virus positive on treatment for 2 years, presented with fatigability and cough was evaluated. He was found to have Retrovirus infection and the insulin requirement increased from 64 units to 162 units per day. He was initiated on multiple doses of human regular insulin. Insulin requirement was high to begin with, and decreased after a few weeks, once glucotoxicity is corrected and infection controlled. Insulin requirements may rise as appetite returns to normal and caloric intake increases.

The purpose of this case report is to emphasize the need to recognize insulin resistance in the presence of chronic liver disease and retroviral infection and to plan Glycemic control accordingly. There is increased incidence of cirrhosis of liver of varying etiology in diabetic patients. The incidence of glucose intolerance varies from 60 to $96 \%$ and that of overt diabetes between 20 and $60 \%$ and is mainly due to insulin resistance [3]. The patient with cirrhosis and diabetes have

\footnotetext{
C. Manjunath $(\bowtie) \cdot$ C. S. Munichoodappa

Department of Diabetololgy, The Bangalore Hospital,

\#202,RV ROAD, Bangalore, Karnataka, India 560004

e-mail: chethana_dr2002@yahoo.com

C. S. Munichoodappa

e-mail: dr.munichoodappa@gmail.com
}

more frequent complications with poor prognosis.Insulin is the treatment of choice and oral agents are contraindicated.In patients with compensated cirrhosis,requirement is greater compared to patients with decompensated cirrhosis, since insulin resistance predominates in the former while in the latter liver metabolism of insulin is greatly reduced [4]. Human immunodeficiency virus (HIV) patients may also present with metabolic syndrome, and lipodystrophy due to adipoknes. Autoimmune diabetes, may develop in some patients after immune restoration during highly active antiretroviral therapy (HAART) especially with PIs as well as nucleoside analogs (reverse transcriptase inhibitors) which cause metabolic dysfunction. Use of opiates and heroin addiction may further compramise beta cell function. Insulin is the drug of choice for management of diabetes in patients inflicted with HIV [5].

Conflicts of interest None

\section{References}

1. Kahn BB. Jeffrey S. Flier Obesity and insulin resistance. 106(4). J Clin Invest. 2000;106:473-81.

2. Castellares C, Barreiro P, Martín-Carbonero L, Labarga P, Vispo ME, Casado R, et al. Liver cirrhosis in HIV-infected patients: prevalence, aetiology and clinical outcome. J Viral Hepat. 2008;15: 165-72.

3. El-Serag HB, Tran T, Everhart JE. Diabetes increases the risk of chronic liver disease and hepatocellular carcinoma. Gastroenterology. 2004; $126: 460-8$.

4. Sanjay K, Bharti K. Navneet Agrawal and AG Unnikrishnan: Understanding diabetes in patients with HIV/AIDS. Diabetol Metab Syndr. 2011;3:2.

5. Norris A, Dreher HM. Lipodystrophy syndrome: the morphologic and metabolic effects of antiretroviral therapy in HIV infection. J Assoc Nurses AIDS Care. 2004;15:46. 